\title{
Cost-Effectiveness Analysis of Cannabinoid Oromucosal Spray Use for the Management of Spasticity in Subjects with Multiple Sclerosis
}

\author{
Lorenzo G. Mantovani ${ }^{1,2} \cdot$ Paolo Cozzolino ${ }^{2} \cdot$ Paolo A. Cortesi ${ }^{1,2}$ (D) Francesco Patti ${ }^{3}$ on behalf of the SA.FE. study \\ group
}

(c) Springer Nature Switzerland AG 2020

\begin{abstract}
Introduction Multiple sclerosis (MS) is a highly symptomatic disease, with a wide range of disabilities affecting many bodily functions, even in younger persons with a short disease history. The availability of a cannabinoid oromucosal spray (Sativex) for the management of treatment-resistant MS spasticity has provided a new opportunity for many patients.

Objective Our study aimed to assess the cost effectiveness of Sativex in Italian patients with treatment-resistant MS spasticity. The analysis was based on the real-world data of a large registry of Italian patients.

Methods A cost-utility analysis was conducted using data collected prospectively from an electronic registry of all patients who began to use Sativex for MS-resistant spasticity between January 2014 and February 2015 in 30 specialized MS units across Italy and were followed up for $\leq 6$ months. Data on drug consumption and spasticity/utility were used to estimate the incremental cost-effectiveness ratio (ICER) of Sativex, as compared with no intervention. No costs or spasticity/utility changes were assumed for no treatment intervention. The ICER was expressed as quality-adjusted life-years (QALYs) gained, using the Italian NHS perspective and a 6-month time horizon.

Results Sativex effectiveness and consumption was estimated analyzing data of 1350 patients from the registry. These patients reported a mean (SD) utility increment of 0.087 (0.069) after 1 month of treatment, $0.118(0.073)$ after 3 months' treatment and $0.127(0.080)$ after 6 months' treatment. The 6-month cost of treating the entire population with Sativex was $€ 1,361,266$, with a $€ 1008$ cost and 0.0284 QALYs gained per patient. The estimated ICER was $€ 35,516$ per QALY gained, with little variability around the central estimate of cost-effectiveness, as shown by the cost-effectiveness acceptability curve. Conclusion The use of Sativex could improve the quality of life of patients with a reasonable incremental cost resulting as a cost-effective option for patients with MS-resistant spasticity. These results could help clinicians and decision makers to develop improved management strategies for spasticity in patients with MS, optimizing the use of available resources.
\end{abstract}

The Members of the SA.FE. study group are listed in acknowledgements section.

Electronic supplementary material The online version of this article (https://doi.org/10.1007/s40261-020-00895-6) contains supplementary material, which is available to authorized users.

Paolo A. Cortesi

paolo.cortesi@unimib.it

1 Research Centre on Public Health (CESP), University of Milano Bicocca, Villa Serena, Via Pergolesi 33, 20900 Monza, Italy

2 Fondazione Charta, Milan, Italy

3 Department of Medical, Surgical Science and Advanced Technology "GF Ingrassia”, University of Catania, Catania, Italy

\section{Introduction}

Multiple sclerosis (MS) is a chronic disease with a variable course and severity; it is one of the most common neurological disorders and affects mainly young adults when work and social relationships are the main aspects of their lives [1-3]. Further, MS is associated with a mean life expectancy of 40 years from diagnosis to the development of significant levels of disability $[1,4]$.

In a recent study, MS reported a high social and economic burden [5]. Costs related to MS coincided with disease severity (disability score), and the main driver was related to productivity loss, non-healthcare costs, and treatment with disease-modifying therapies (DMTs) [5-7]. The mean 


\section{Key Points}

Our study provided empirical evidence of Sativex cost effectiveness based on real-world data.

The use of Sativex could improve the quality of life of patients with a reasonable increment of costs

Sativex demonstrated an ICER of $€ 35,516$ per QALY gained, resulting as a cost-effective intervention in patients with MS-resistant spasticity.

costs per patient-year for mild, moderate, and severe MS were $€ 22,800$; $€ 37,100$; and $€ 57,500$, respectively; as previously mentioned, the loss of productivity was the main cost, with the proportion of unemployed patients around $50 \%$, if considering only working-age patients [5].

The availability of DMTs since 2000 has had a great impact on the management of MS, reducing disease progression [8]. Further, improved diagnostic criteria have enabled earlier diagnosis and treatment [9]. This has led to costs shifting, and now, DMTs represent the main cost driver for patients with a low level of disability [5]. However, DMTs have reduced inflammatory activity and slowed disease progression but with no impact on existing impairments; consequently, most of the management for patients with MS must focus on optimizing function and controlling symptoms [3, $10,11]$. Indeed, MS is a highly symptomatic disease with a wide range of disabilities affecting many bodily functions, even in younger persons (aged $\leq 35$ years), with a short disease history $[12,13]$. Appropriate symptom management is essential to improve rehabilitation and to promote well-being $[3,13,14]$.

The availability of 9- $\delta$-tetrahydrocannabinol and cannabidiol (THC:CBD) oromucosal spray (Sativex) for the management of treatment-resistant MS spasticity opened a new opportunity for many patients [4, 15-17]. Our study aimed to assess the cost effectiveness of Sativex in Italian patients with treatment-resistant MS spasticity. The analysis was based on the real-world data of a large registry of Italian patients.

\section{Methods}

This study compared the Sativex treatment with "no treatment" option in MS patients with resistant spasticity. The no treatment comparator was selected based on the lack of other effective treatment for MS patients with resistant spasticity.
To reach the objective of the study, we assessed the Sativex treatment efficacy and consumption in MS patients with resistant spasticity from the Italian Sativex Registry. This is a mandatory registry established by the Italian Medicines Agency (AIFA) to verify the effectiveness and tolerability of Sativex in clinical practice and to apply the payment by results and cost-sharing agreement between the manufacturer and the Italian National Health Service (NHS). All patients treated in Italy with Sativex must be included in the Registry, otherwise the Italian NHS not pay for the treatment.

Patients were consecutively included in the registry at the start of Sativex treatment (baseline) and followed up over 6 months, with data collection at baseline, and after 4, 12 and 24 weeks [16]. The registry reported demographical and clinical history data, MS spasticity [0-10 numerical rating scale (NRS)] data, MS physical disability [Expanded Disability Status Scale (EDSS)], Sativex treatment (tolerability, daily dose/number of puffs per day, clinical response and discontinuation).

Based on the Sativex registry follow-up, we performed a cost-effectiveness analysis using a 6-month time horizon from the Italian NHS perspective and a cost $(€)$ per qualityadjusted life year (QALY) gained as outcome. No costs and outcome discount rates were applied due to the time horizon shorten than 1 year.

\subsection{Population}

From the mandatory AIFA prospective electronic registry, we retrieved data for all patients who began using Sativex for MS-resistant spasticity between January 2014 and February 2015 in 30 specialized MS units across Italy and were followed up for $\leq 6$ months.

The inclusion criteria in the AIFA registry was patients with MS, aged $>18$ years, from moderate-to-severe spasticity (NRS score $\geq 4$ ) and not responding to common and ongoing antispastic drugs [16]. Exclusion criteria were severe cardiovascular diseases, history of psychiatric diseases, use of street cannabis and/or other psychoactive drugs [16].

\subsection{Effects}

The analysis compared two treatments option: (1) Sativex treatment and (2) No treatment. The treatment effectiveness was reported as QALYs, estimated based on the correlation ("mapping") between utility value and the NRS score.

The Sativex treatment effectiveness was estimated, analyzing the 1-month, 3-month and 6-month NRS scores reported by the patients in the AIFA registry. For the "No treatment" option, we applied the NRS score estimated at baseline in the AIFA registry to all 6-month periods. This 
is a reasonable approach because with no expected clinical improvement considering the resistance shown to the previous treatments by the patients included in the registry and the lack of other effective intervention other than Sativex.

The NRS scores estimated from the AIFA registry was transformed in utility value following the correlation ("mapping") between EQ-5D utility value and the NRS score reported by Svensson and colleagues [18]. This study was selected because it reported the most robust methodological approach in the literature estimating the regression coefficients adjusting for age, sex, and EDSS (data available in our registry), thus providing the net expected effect of changes in utility associated with changes in the spasticity NRS.

The use of "mapping" techniques to link the outcomes from different measures of health or health-related quality of life (HRQOL) is a well-established and frequently used approach in health economics evaluations, especially in costutility analyses of health technologies [19-22]. These methods are useful when the studies estimate the effectiveness of interventions without collecting health-related utility data [20-22]. In addition, when we need the utility values estimated by a specific preference-based measure, the mapping approach can be used to link to health-related utility values for that instrument by using outcomes obtained from other measures/instruments [20, 21]. An example is provided by the United Kingdom, where the National Institute for Health and Clinical Excellence (NICE) accept to map to, or predict, the health-related utility values of EQ-5D data, even where other measures have been collected [20,22].

To estimate QALYs in our study, we partitioned observation time into intervals according to the spasticity NRS assessment points. We calculated QALYs gained as the product of time (between two assessments) and the change in utility associated with a change in the spasticity NRS adjusting for the patient's EDSS (estimated using the coefficient of Svensson and colleagues) [18]. To calculate time appropriately, we used the actual recorded time between the two assessments and assumed the change in the spasticity NRS (and consequently in utility) to be linear. Svensson and colleagues estimated that there would be a utility gain of 0.0505 for each decrease in a spasticity NRS point. The full equation used to estimate the utility at each time point was:

$$
\begin{aligned}
\text { Utility }= & 0.9229-\operatorname{NRS} \times(-0.0505)-0.0293(\text { EDSS 5.0) } \\
& -0.3417(\text { EDSS 5.5) }-0.1305(\text { EDSS 6.0) } \\
& -0.2521(\text { EDSS 6.5) }-0.3353(\text { EDSS 7.0) } \\
& -0.5260(\text { EDSS 7.5) }-0.8124(\text { EDSS } 8.0) \\
& -0.9408(\text { EDSS } 8.5)-0.7648(\text { EDSS 9.0) }
\end{aligned}
$$

We cumulated all QALY gained during each period to compute the overall per-patient QALY gained. After treatment discontinuation, we assumed no further utility gains. Treatment discontinuation was assumed, for each patient, after the last visit with efficacy data reported. The approach selected gave the possibility to link the treatment consumption to its efficacy.

\subsection{Cost}

We computed costs from the perspective of the Italian NHS and expressed them in Euros (€2017). Our analysis was limited to drug costs, based on the number of puffs documented in the registry. The ex-factory cost for a puff of Sativex was $€ 1.47$, and this cost did not include the initial treatment costs of the non-responder patients based on the access scheme for Italian patients (payment by results agreement between the Italian Medicines Agency and the manufacturer) [23]. For the "No treatment" intervention we assumed a cost of $€ 0$, because no treatment was provided.

Other healthcare costs were not computed, as they were not collected in the AIFA registry. As for increased healthcare and social costs incurred by patients with more severe spasticity, our assumption can be considered conservative based on the NRS improvement associated only with Sativex treatment [18]. After treatment discontinuation, we assumed no further costs.

\subsection{Cost-Effectiveness Analysis}

For every single patient included in the AIFA registry, we computed the treatment cost and QALYs as the sum of costs incurred during the observation time, and the improvement in NRS score expressed as QALYs. For the "No treatment" option we assumed a 6-month cost of $€ 0$ and no utility change compared to the value estimate at baseline in the AIFA registry. The difference in costs and QALYs between the two treatment options included in our analysis was used to assess the cost effectiveness estimating the ICER expressed as $€$ per QALY gained.

\subsection{Sensitivity Analysis}

The uncertainty in our analysis was related to the costs and QALY gained associated with the Sativex treatment and estimated for each patient using the AIFA registry data. Based on the availability of patient level data, we calculated the cost-effectiveness acceptability curve (CEAC) using standard sampling-resampling bootstrapping techniques to include the variability associated with the ICER reported by the population included in the study as performed by Olivieri et al $[24,25]$. To estimate the CEAC, we also included the parameter uncertainty of Svenson et al mapping equation; the standard errors associated with each equation coefficient are reported in Supplementary Table 1. This analysis was performed sampling 1000 times the equation coefficients, assuming a normal distribution for all coefficients, and 
Table 1 Clinical and demographic data of cohort included in the cost-effectiveness analysis $(N=1350)$

\begin{tabular}{ll}
\hline Parameter & Value \\
\hline Male $(n, \%)$ & $635(47.0)$ \\
Female $(n, \%)$ & $715(53.0)$ \\
Age (years, mean \pm SD) & $50.9(9.6)$ \\
Disease duration (years, mean \pm SD) & $17.7(8.6)$ \\
Baseline EDSS (median, IQR) & $6.5(6.0-7.0)$ \\
NRS score T0, baseline (mean, SD) & $7.5(1.5)$ \\
NRS score T1, baseline (mean, SD) & $5.8(1.6)$ \\
NRS score T2, baseline (mean, SD) & $5.1(1.6)$ \\
NRS score T3, baseline (mean, SD) & $4.8(1.7)$ \\
Dose, puffs number T1 (mean, SD) & $6.8(2.5)$ \\
Dose, puffs number T2 (mean, SD) & $6.5(2.6)$ \\
Dose, puffs number T3 (mean, SD) & $6.3(2.8)$ \\
\hline
\end{tabular}

EDSS expanded disability status scale, IQR interquartile range, $N R S$ numerical rating scale, $S D$ standard deviation

performing the sampling-resampling bootstrapping for each of the 1000 value of Svenson et al equation coefficients.

\section{Results}

Of the 1615 patients included in the AIFA registry, 1350 were included in our analysis to estimate the Sativex costs and effectiveness. Two hundred sixty-five patients were excluded because they did not report information on the treatment efficacy and/or consumption. The mean (SD) age was 50.9 (9.6), and $47.0 \%$ were male (Table 1). A difference was found while comparing the baseline score with the NRS score values determined at different time points for the remaining patients. The mean (SD) NRS score was $7.5(1.5)$ at $\mathrm{T} 0,5.8(1.6)$ at $\mathrm{T} 1,5.1(1.6)$ at $\mathrm{T} 2$, and $4.8(1.7)$ at $\mathrm{T} 3$ (Table 1).

A mean (SD) utility increment of 0.087 (0.069) was reported at T1 $(n=1350)$ (Fig. 1). The 3-months visit (T2) was performed by 853 patients, with a utility increment of 0.118 (0.073) compared to T0. While the 6-month visit (T3) was achieved by 558 patients, with a utility increment of 0.127 (0.080) compared to T0 (Fig. 1).

The analysis of the medication consumption showed a mean (SD) number of puffs per day of 6.8 (2.6) at T1 (Table 1). A reduction of medication consumption was reported at $\mathrm{T} 2$ and $\mathrm{T} 3$, with a mean (SD) number of puffs per day of $6.5(2.6)$ and $6.3(2.8)$ respectively (Table 1). The analysis of the Staivex treatment showed a mean (SD) cost per patient-month of $€ 302.0$ (111.1) at T1, with a slightly reduction to $€ 294.0$ (109.0) at $\mathrm{T} 2$, and to $€ 288.1$ (111.4) at T3. The majority of patients reporting a monthly cost between $€ 250$ and $€ 350$ in all 3 time periods of follow-up (Fig. 2). The overall cost of treating the entire population with Sativex was $€ 1,361,266$ during the 6 months of the study, and the total number of QALYs was 235.4 for the whole population treated. During the 6-month analysis, the mean (SD) estimated cost was $€ 1008.3$ (378.8) per patient and $0.1744(0.0658)$ QALYs (Table 2). In the "No treatment" option, the 6-month cost was $€ 0$ per patient and the mean (SD) estimated QALY was 0.1460 (0.0551). Based on the Sativex and "No treatment" QALYs and cost differences, the cost-effectiveness analysis provided an ICER of $€ 35,516$ per QALY gained, with little variability around the central estimate of cost effectiveness, as shown by the costeffectiveness acceptability curve (Fig. 2). The probability of Sativex to be the cost-effective option at a willingness
Fig. 1 Utility changes during follow-up. Comparison between baseline utility value and each follow-up visit. $C I$ confidence interval, $\Delta \mathrm{T} 1-\mathrm{T} 0$ difference between $\mathrm{T} 1$ and $\mathrm{T} 0$ utility values; $\Delta \mathrm{T} 2-\mathrm{T} 0$ difference between $\mathrm{T} 2$ and $\mathrm{T} 0$ utility values; $\Delta$ T3-T0 difference between $\mathrm{T} 3$ and $\mathrm{T} 0$ utility values

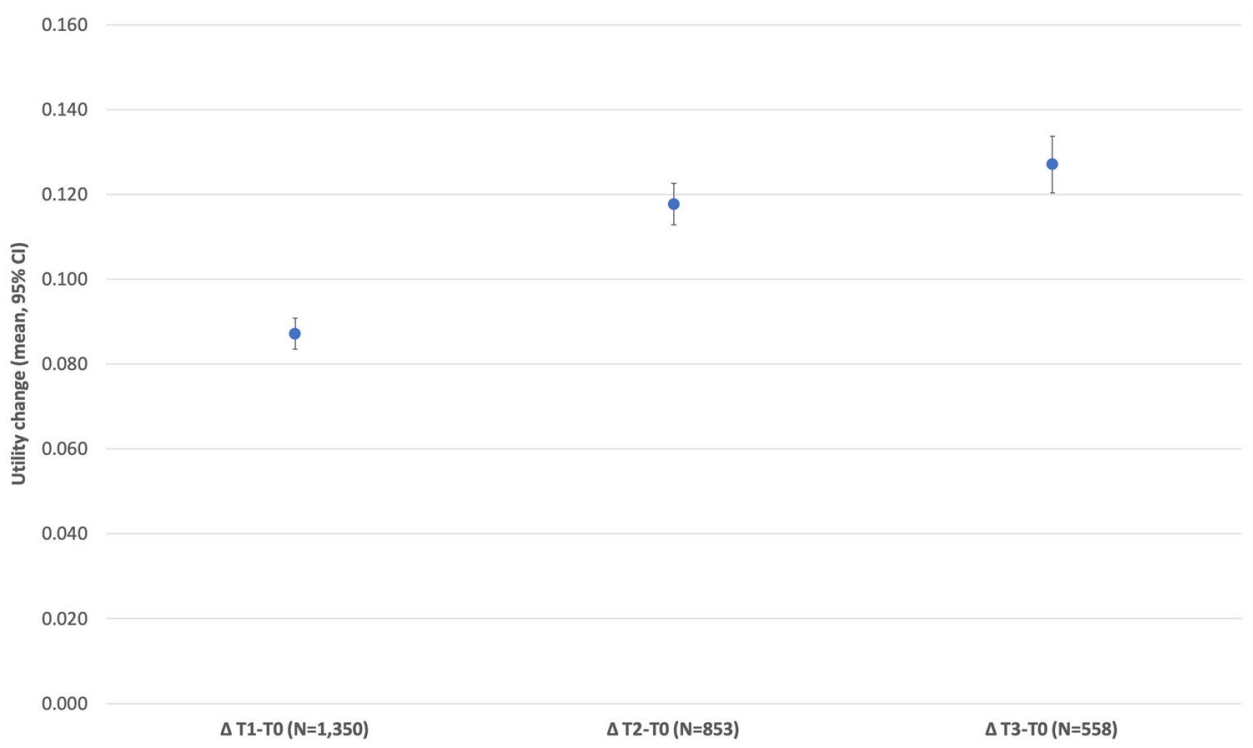




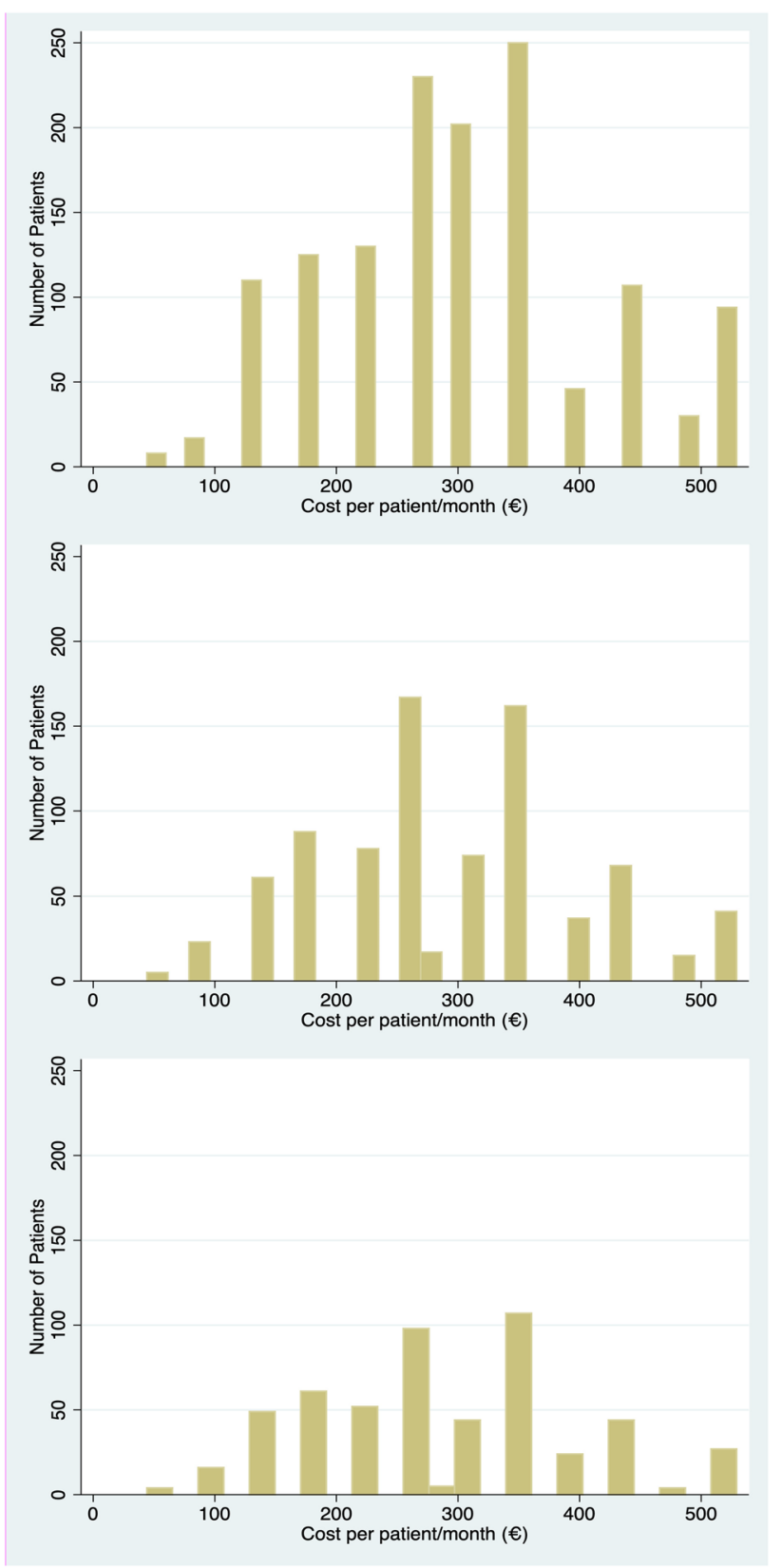

Fig. 2 Cost per patient-month distribution during the time between the follow-up visits: a T0-T1, b T1-T2, and c T2-T3 to pay (WTP) threshold of $€ 40,000$ per QALY gained was virtually $100 \%$.

\section{Discussion}

MS is associated with an early age of onset and a significant impact on HRQoL [6, 8]. This condition requires life-long, coordinated treatment and management $[6,8]$. The chronic and degenerative characteristics of MS produced substantial economic burden on individuals, healthcare systems, and society $[5,6]$. Within the relevant social and economic impact of MS symptoms, spasticity is associated with high costs, with annual costs per patient that increase with the levels of severity $[2,18,26,27]$. Key factors associated with high annual costs per patient with MS and spasticity are (1) home care costs; (2) hospital admissions; and (3) high cost items [3, 28].

Our study showed, in real clinical practice, how the use of Sativex could improve the management of patients with MS and resistant spasticity, resulting in a cost-effective intervention. Based on the cost-effectiveness analysis results, Sativex reported an ICER of $€ 35,516$ per QALY gained compared to the "No treatment" option, with little variability around the ICER central estimate. Further, the probability of Sativex to be a cost-effective option at a WTP threshold of $€ 40,000$ per QALY gained was virtually $100 \%$. The ICER estimated was under the WTP threshold of $€ 40,000$ per QALY gained that is considered acceptable by leading regulatory agencies such as NICE-UK, AHRQ-USA, and CADTH-Canada [29] and is under the WTP of $€ 60,000$ per QALY gained used in Italy $[25,30]$.

Previous model-based cost-effectiveness studies reported extremely diverse results, with Sativex cost-effectiveness profile ranging from cost savings to no cost-effective savings [31]. A recent review of the cost and benefits of cannabisbased medicines for the management of MS reported that Sativex was a cost-effective option in four of the five studies retrieved. These studies were based on decision analytical model simulations and were conducted using healthcare setting from Spain [32], Germany [32], Italy [33], Wales [34], and UK [35] healthcare settings. In the studies where Sativex resulted in cost effectiveness, the ICERs reported were $€ 4968$ (Italy), £10,891 (Wales), and €11,214 (Germany)

Table 2 Cost-effectiveness analysis results. Costs and QALYs are reported per patient-6 months

\begin{tabular}{lllll}
\hline Treatment & Total costs $(€)$ & $\Delta$ Total costs $(€)$ & QALYs & $\Delta$ QALYs $^{\text {ICER }^{\mathrm{a}}}$ \\
\hline No treatment & 0 & - & 0.1460 & - \\
Sativex & 1008.34 & 1008.34 & 0.1744 & 0.0284 \\
\hline
\end{tabular}

ICER incremental cost-effectiveness ratio, $Q A L Y$ quality-adjusted life-year

${ }^{\mathrm{a}}$ ICER reported as $€$ per QALY gained 
per QALY gained and dominant (less expensive and more effective) in the Spanish analysis. The study conducted in the UK reported an ICER of $£ 49,257$, with Sativex considered not cost effective. Apart from applying the different costs to each healthcare system for the different elements considered, discrepancies were mainly attributable to the models' assumptions (effect onset, effect duration, number of puffs per day, etc.) that is understandable due to the scarce or lack of observations. Our study provided real-world evidence on Sativex cost effectiveness for patients with MS who have moderate-to-severe spasticity that is resistant to common anti-spasticity drugs (Fig. 3).

Our cost-effectiveness analysis was conducted on realworld patient-level data from a national registry, and it was not based on decision analytical model simulations. The choice of not performing an analysis based on a decision model, that gives the possibility to simulate a longer time horizon, is reasonable considering the type of treatment evaluated. Sativex is indicated to treat spasticity and related symptoms associated with MS and not to modify the progression of the diseases. This treatment reports an effect on patients' spasticity when the drug is taken, with no other relevant effects when the treatment is administrated for a time period as short as 6 months [36]. Based on this consideration, assessing the effect and cost of 6 months' treatment with Sativex was a reliable approach to understand its cost effectiveness in the first period of administration and help clinicians and decision makers to understand the value of starting a treatment with Sativex in patients with treatmentresistant MS spasticity. However, further analyses with clinical and cost data based on a longer follow-up are necessary and helpful to understand the cost effectiveness of Sativex administrated for a longer period.
Our study had some limitations that should be discussed. First, the utility values were estimated based on a mapping analysis [18, 21]. Estimating a utility value based on data collected directly from the EQ-5D questionnaire is the most robust approach. However, using indirect estimations of utility value based on a mapping approach is a well-established, reliable and accepted method [20-22]. Further, the data used for our analysis were the best found in the literature to estimate a utility value based on the NRS score, because the regression coefficients calculated in the mapping analysis were simultaneously adjusted for EDSS, providing a net expected effect of changes in utility associated with changes in the spasticity NRS score. However, other MS symptoms not included in the regression analysis would have biased the relationship between spasticity and HRQoL in the mapping analysis. Secondly, we included only the cost associated with Sativex. This apprach can be considered conservative due to the higher cost (home care cost, hospital admissions, and high-cost items) associated with MS patients with uncontrolled and severe spasticity [3,28]. Considering the Sativex efficacy in controlling and reducing spasticity, including the other direct costs could made the cost effectiveness of Sativex even better. Finally, in the study we assumed no costs or utility value change for the "No treatment" alternative option, as done in similar previous studies conducted in other medical condition (e.g. psoriasis, psoriatic arthritis) [25]. The assumption of no reduction in utility value for "No treatment" option was a conservative approach; considering that the population included in the study was patients with uncontrolled and resistant MS spasticity.
Fig. 3 Cost-effectiveness acceptability curve for Sativex. $W T P$ willingness to pay in $€$

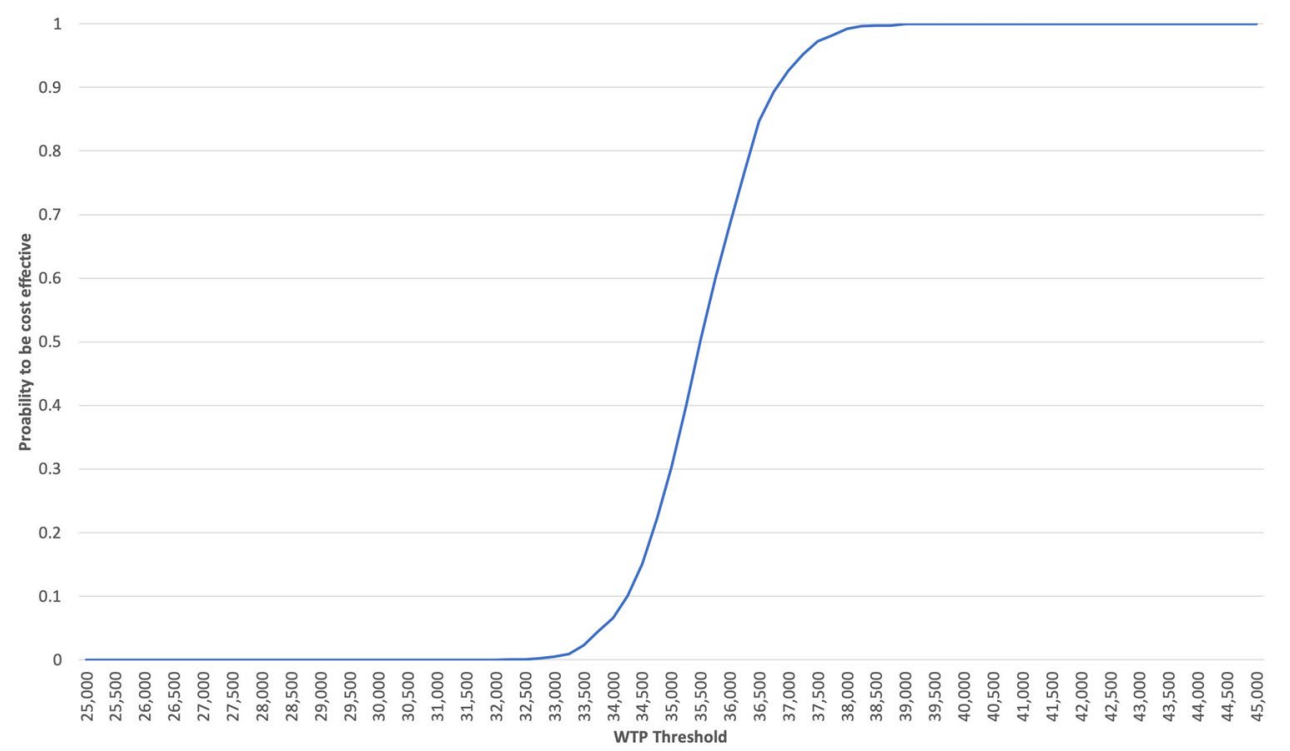




\section{Conclusion}

Patients with MS who have moderate-to-severe spasticity experience high economic and societal issues. The use of Sativex could improve the spasticity control and severity, providing a better quality of life in these patients with a reasonable increment of costs. Our analysis showed the cost effectiveness of Sativex in patients with MS-resistant spasticity in the first 6 months of treatment. These results may help clinicians and decision makers in implementing more effective management strategies for MS-associated spasticity, optimizing the use of available resources. New economic analyses, using data collected over a longer time horizon and including all costs data, are needed to show the cost effectiveness of long-term treatment with Sativex.

Acknowledgements The authors would like to thank BioMed Proofreading ${ }^{\circledR}$ for the support in copyediting the manuscript. The Members of the SA.FE. study group: Patti F, Messina S, Solaro C, Amato MP, Bergamaschi R, Bonavita S, Bruno Bossio R, Brescia Morra V, Costantino GF, Cavalla P, Centonze D, Comi G, Cottone S, Danni M, Francia A, Gajofatto A, Gasperini C, Ghezzi A, Iudice A, Lus G, Maniscalco GT, Marrosu MG, Matta M, Mirabella M, Montanari E, Pozzilli C, Rovaris M, Sessa E, Spitaleri D, Trojano M, Valentino P, Zappia M, Benedetti MD, Bertolotto A, Berra E, Bianco A, Buttari F, Cerqua R, Florio C, Fuiani A, Guareschi A, Ippolito D, Nuara A, Palmieri V, Paolicelli D, Petrucci L, Pontecorvo S, Saccà F, Salomone G, Signoriello E, Spinicci G, Russo M, Tavazzi E, Trabucco E, Trotta $\mathrm{M}$, Zaffaroni M.

Author Contributions Conceptualization, LGM and FP; methodology, LGM, PC, and PAC; data curation: PC; formal analysis, PC and PAC; writing Sativex original draft preparation, PAC and PC; writing Sativex review and editing, LGM and FP; funding acquisition: LGM.

\section{Compliance with Ethical Standards}

Funding This study was supported by an unrestricted research grant from Almirall S.p.A.

Conflict of interest PAC received a research grant from Baxalta now part of Shire and speaking honoraria from Pfizer and Roche in areas other than Multiple Sclerosis; L.G.M. has received grants from MSD and Janssen and honoraria from Bayer in areas other than Multiple Sclerosis. F.P. received honoraria for speaking activities and/or serving as advisor board member by Almirall, Bayer, Biogen, Celgene, Merck, Myalin, Novartis, Roche, Sanofi-Genzyme, and TEVA. He was also granted for research projects by MIUR, University of Catania, FISM and ISS. P.C. have nothing to declare.

\section{References}

1. Compston A, Coles A. Multiple sclerosis. Lancet. 2002;359(9313):1221-31.

2. Feinstein A, Freeman J, Lo AC. Treatment of progressive multiple sclerosis: what works, what does not, and what is needed. Lancet Neurol. 2015;14(2):194-207.
3. Stevenson VL, Gras A, Bárdos JI, Broughton J. The high cost of spasticity in multiple sclerosis to individuals and society. Mult Scler. 2015;21(12):1583-92.

4. Messina S, Solaro C, Righini I, Bergamaschi R, Bonavita S, Bossio RB, Brescia Morra V, Costantino G, Cavalla P, Centonze D, Comi G, Cottone S, Danni MC, Francia A, Gajofatto A, Gasperini C, Zaffaroni M, Petrucci L, Signoriello E, Maniscalco GT, Spinicci G, Matta M, Mirabella M, Pedà G, Castelli L, Rovaris M, Sessa E, Spitaleri D, Paolicelli D, Granata A, Zappia M, Patti F, S. F. s. group. Sativex in resistant multiple sclerosis spasticity: discontinuation study in a large population of Italian patients ( $\mathrm{Sa}$. Fe. study). PLoS One. 2017;12(8):e0180651.

5. Kobelt G, Thompson A, Berg J, Gannedahl M, Eriksson J, M. S. Group and E. M. S. Platform. New insights into the burden and costs of multiple sclerosis in Europe. Mult Scler. 2017;23(8):1123-36.

6. Battaglia M, Kobelt G, Ponzio M, Berg J, Capsa D, Dalén J, E. M. S. Platform. New insights into the burden and costs of multiple sclerosis in Europe: results for Italy. Mult Scler. 2017;23(2):104-16.

7. Salter AR, Cutter GR, Tyry T, Marrie RA, Vollmer T. Impact of loss of mobility on instrumental activities of daily living and socioeconomic status in patients with Ms. Curr Med Res Opin. 2010;26(2):493-500.

8. Capra R, Cordioli C, Rasia S, Gallo F, Signori A, Sormani MP. Assessing long-term prognosis improvement as a consequence of treatment pattern changes in Ms. Mult Scler. 2017;23(13):1757-61.

9. Thompson AJ, Banwell BL, Barkhof F, Carroll WM, Coetzee T, Comi G, Correale J, Fazekas F, Filippi M, Freedman MS, Fujihara K, Galetta SL, Hartung HP, Kappos L, Lublin FD, Marrie RA, Miller AE, Miller DH, Montalban X, Mowry EM, Sorensen PS, Tintoré M, Traboulsee AL, Trojano M, Uitdehaag BMJ, Vukusic S, Waubant E, Weinshenker BG, Reingold SC, Cohen JA. Diagnosis of multiple sclerosis: 2017 revisions of the Mcdonald criteria. Lancet Neurol. 2018;17(2):162-73.

10. Rønning OM, Tornes KD. Need for symptomatic management in advanced multiple sclerosis. Acta Neurol Scand. 2017;135(5):529-32.

11. Skierlo S, Rommer PS, Zettl UK. Symptomatic treatment in multiple sclerosis-interim analysis of a Nationwide Registry. Acta Neurol Scand. 2017;135(4):394-9.

12. Heesen C, Böhm J, Reich C, Kasper J, Goebel M, Gold SM. Patient perception of bodily functions in multiple sclerosis: gait and visual function are the most valuable. Mult Scler. 2008;14(7):988-91.

13. Patti F, Vila C. Symptoms, prevalence and impact of multiple sclerosis in younger patients: a multinational survey. Neuroepidemiology. 2014;42(4):211-8.

14. Arroyo R, Massana M, Vila C. Correlation between spasticity and quality of life in patients with multiple sclerosis: the candle study. Int J Neurosci. 2013;123(12):850-8.

15. Novotna A, Mares J, Ratcliffe S, Novakova I, Vachova M, Zapletalova O, Gasperini C, Pozzilli C, Cefaro L, Comi G, Rossi P, Ambler Z, Stelmasiak Z, Erdmann A, Montalban X, Klimek A, Davies P. A randomized, double-blind, placebo-controlled, parallel-group, enriched-design study of nabiximols* (Sativex $\left({ }^{\circledR}\right)$ ), as add-on therapy, in subjects with refractory spasticity caused by multiple sclerosis. Eur J Neurol. 2011;18(9):1122-31.

16. Patti F, Messina S, Solaro C, Amato MP, Bergamaschi R, Bonavita S, Bruno Bossio R, Brescia Morra V, Costantino GF, Cavalla P, Centonze D, Comi G, Cottone S, Danni M, Francia A, Gajofatto A, Gasperini C, Ghezzi A, Iudice A, Lus G, Maniscalco GT, Marrosu MG, Matta M, Mirabella M, Montanari E, Pozzilli C, Rovaris M, Sessa E, Spitaleri D, Trojano M, Valentino P, Zappia M. Efficacy and safety of cannabinoid oromucosal spray for 
multiple sclerosis spasticity. J Neurol Neurosurg Psychiatry. 2016;87(9):944-51.

17. Vermersch P, Trojano M. Tetrahydrocannabinol: cannabidiol oromucosal spray for multiple sclerosis-related resistant spasticity in daily practice. Eur Neurol. 2016;76(5-6):216-26.

18. Svensson J, Borg S, Nilsson P. Costs and quality of life in multiple sclerosis patients with spasticity. Acta Neurol Scand. 2014;129(1):13-20.

19. Brazier JE, Yang Y, Tsuchiya A, Rowen DL. A review of studies mapping (or cross walking) non-preference based measures of health to generic preference-based measures. Eur J Health Econ. 2010;11(2):215-25.

20. Longworth L, Rowen D. Mapping to obtain Eq-5d utility values for use in nice health technology assessments. Value Health. 2013;16(1):202-10.

21. Wailoo AJ, Hernandez-Alava M, Manca A, Mejia A, Ray J, Crawford B, Botteman M, Busschbach J. Mapping to estimate healthstate utility from non-preference-based outcome measures: an ISPOR good practices for outcomes research task force report. Value Health. 2017;20(1):18-27.

22. Wolowacz S. New ISPOR recommendations-mapping methods for estimation of health state utility. Value Health. 2017;20(1):28-9.

23. AIFA. Procedura Di Applicazione, Managed Entry Agreement Medicinale Sativex. 2017. https://www.aifa.gov.it/-/procedura-diapplicazione-managed-entry-agreement-medicinale-sativex-0103-2017-. Accessed 24 Feb 2020.

24. Fenwick E, O'Brien BJ, Briggs A. Cost-effectiveness acceptability curves-facts, fallacies and frequently asked questions. Health Econ. 2004;13(5):405-15.

25. Olivieri I, de Portu S, Salvarani C, Cauli A, Lubrano E, Spadaro A, Cantini F, Cutro MS, Mathieu A, Matucci-Cerinic M, Pappone N, Punzi L, Scarpa R, Mantovani LG. The psoriatic arthritis cost evaluation study: a cost-of-illness study on tumour necrosis factor inhibitors in psoriatic arthritis patients with inadequate response to conventional therapy. Rheumatology. 2008;47(11):1664-70.

26. Berger T. Multiple sclerosis spasticity daily management: retrospective data from Europe. Expert Rev Neurother. 2013;13(3 Suppl 1):3-7.
27. Zettl UK, Henze T, Essner U, Flachenecker P. Burden of disease in multiple sclerosis patients with spasticity in Germany: mobility improvement study (Move I). Eur J Health Econ. 2014;15(9):953-66.

28. Pozzilli C. Advances in the management of multiple sclerosis spasticity: experiences from recent studies and everyday clinical practice. Expert Rev Neurother. 2013;13(12 Suppl):49-54.

29. Cortesi PA, Mantovani LG, Ciaccio A, Rota M, Mazzarelli C, Cesana G, Strazzabosco M, Belli LS. Cost-effectiveness of new direct-acting antivirals to prevent post-liver transplant recurrent hepatitis. Am J Transplant. 2015;15(7):1817-26.

30. Cortesi PA, D’Angiolella LS, Vellucci R, Allegri M, Casale G, Favaretti C, Kheiraoui F, Cesana G, Mantovani LG. Cost-effectiveness analysis of oral fentanyl formulations for breakthrough cancer pain treatment. PLoS One. 2017;12(6):e0179523.

31. Herzog S, Shanahan M, Grimison P, Tran A, Wong N, Lintzeris N, Simes J, Stockler M, Morton RL. Systematic review of the costs and benefits of prescribed cannabis-based medicines for the management of chronic illness: lessons from multiple sclerosis. Pharmacoeconomics. 2018;36(1):67-78.

32. Slof J, Gras A. Sativex ${ }^{\circledR}$ in multiple sclerosis spasticity: a costeffectiveness model. Expert Rev Pharmacoecon Outcomes Res. 2012;12(4):439-41.

33. Slof J, Ruiz L, Vila C. Cost-effectiveness of Sativex in multiple sclerosis spasticity: new data and application to Italy. Expert Rev Pharmacoecon Outcomes Res. 2015;15(3):379-91.

34. Gras A, Broughton J. A cost-effectiveness model for the use of a cannabis-derived oromucosal spray for the treatment of spasticity in multiple sclerosis. Expert Rev Pharmacoecon Outcomes Res. 2016;16(6):771-9.

35. Lu L, Pearce H, Roome C, Shearer J, Lang IA, Stein K. Cost effectiveness of oromucosal cannabis-based medicine (Sativex ${ }^{\circledR}$ ) for spasticity in multiple sclerosis. Pharmacoeconomics. 2012;30(12):1157-71.

36. Oreja-Guevara C, Casanova B, Ordás CM, Vila C, et al. Observational safety study of Thc: Cbd Oromucosal Spray (Sativex) in multiple sclerosis patients with spasticity. Clin Exp Pharmacol. 2015;5:184. 